\title{
Context Aware Hybrid Agents on Automated Dynamic Environments
}

\author{
Juan F. de Paz, Sara Rodríguez, Juan M. Sánchez, Ana de Luis and Juan M. Corchado \\ Departamento Informática y Automática, Universidad de Salamanca \\ Plaza de la Merced s/n, 37008, Salamanca, Spain \\ \{fcofds, srg, adeluis, corchado\}@usal.es; darksnaider@hotmail.com
}

\begin{abstract}
This paper presents an Ambient Intelligence based architecture model that uses intelligent hybrid agents with the ability to obtain automatic and real time information about the context using a set of technologies, such as radio frequency identification, wireless networks and wireless control devices, which can be implemented on a wide diversity of dynamic environments.
\end{abstract}

Keywords: Case-based planning; Context Aware; Hybrid systems

\section{Introduction}

Agents and multi-agent systems (MAS) have become increasingly relevant for developing distributed and dynamic open systems, as well as the use of context aware technologies that allow those systems to obtain information about the environment. This paper is focused on describing the main characteristics of an Ambient Intelligence based architecture which integrates deliberative BDI (Believe, Desire, Intention) agents that employ Radio Frequency Identification, wireless networks, and automation devices to provide automatic and real time information about the environment, and allow the users to interact with their surroundings, controlling and managing physical services (i.e. heating, lights, switches, etc.). These context aware agents collaborate with hybrid agents that use Case-Based Reasoning (CBR) and Case-Based Planning (CBP) as reasoning mechanisms as a way to implement adaptive systems on automated dynamic environments.

A hybrid CBR-BDI agent [5] uses Case-Based Reasoning as a reasoning mechanism, which allows it to learn from initial knowledge, to interact autonomously with the environment as well as with users and other agents within the system, and to have a large capacity for adaptation to the needs of its surroundings. We shall refer to the hybrid CBR-BDI agents specialized in generating plans as hybrid CBP-BDI agents. BDI agents can be implemented by using different tools, such as Jadex [14]. Jadex agents deal with the concepts of beliefs, goals and plans; they are java objects that can be created and handled within the agent at execution time. 
The architecture is founded on Ambient Intelligence (AmI) environments, characterized by their ubiquity, transparency and intelligence. Ambient Intelligence proposes a new way to interact between people and technology, where this last one is adapted to individuals and their context, showing a vision where people are surrounded by intelligent interfaces merged in daily life objects [8], creating a computing-capable environment with intelligent communication and processing to the service of people by means of a simple, natural, and effortless human-system interaction for users [16], reason why to develop intelligent and intuitive systems and interfaces, capable to recognize and respond to the users necessities in a ubiquous way [7], considering people in the centre of the development [17], and creating technologically complex environments in medical, domestic, academic, etc. fields. [20]. Agents in this context must be able to respond to events, take the initiative according to their goals, communicate with other agents, interact with users, and make use of past experiences to find the best plans to achieve goals.

Next, the main characteristics of the architecture are explained, focusing on the context aware agents and the technologies integrated to them.

\section{Hybrid Reasoning and Planning Agents}

All agents in this development are based on the BDI (Belief, Desire, Intention) deliberative architecture model [3], where the internal structure and capabilities of the agents are based on mental aptitudes, using beliefs, desires and intentions. We have implemented hybrid agents which integrates CBR systems [1] as a deliberative mechanism within BDI agents, facilitating learning and adaptation and providing a greater degree of autonomy than pure BDI architecture. CBR is a type of reasoning based on the use of past experiences [12] to solve new problems by adapting solutions that have been used to solve similar problems in the past, and learn from each new experience. To introduce a CBR motor into a deliberative BDI agent it is necessary to represent the cases used in a CBR system by means of beliefs, desires and intentions, and then implement a CBR cycle to process them.

The primary concept when working with CBR systems is the concept of case, which is described as a past experience composed of three elements: an initial state or problem description that is represented as a belief; a solution, that provides the sequence of actions carried out in order to solve the problem; and a final state that is represented as a set of goals. CBR manages cases (past experiences) to solve new problems. The way cases are managed is known as the CBR cycle, and consists of four sequential phases: retrieve, reuse, revise and retain. The retrieve phase starts when a new problem description is received. Similarity algorithms are applied in order to retrieve from the cases memory the cases with a problem description more similar to the current one. Once the most similar cases have been retrieved, the reuse phase begins, adapting the solutions for the retrieved cases to obtain the best solution for the current case. The revise phase consists of an expert revision of the solution proposed. Finally, the retain phase allows the system to learn from the experiences obtained in the three previous phases and consequently updates the cases memory. The retrieve and reuse phases are implemented through FYDPS [11] neural networks 
which allow the agent to recover similar cases from the cases memory and to adapt their solutions using supervised learning, in order to obtain a new optimal solution. The incorporation of these neural networks in the reasoning/planning mechanism reinforces the hybrid characteristics of the agent.

In a hybrid planning agent, the reasoning motor generates plans using past experiences and planning strategies, so the concept of Case Base Planning is obtained [9]. CBP consists of four sequential stages: retrieve stage to recover the most similar past experiences to the current one; reuse stage to combine the retrieved solutions in order to obtain a new optimal solution; revise stage to evaluate the obtained solution; and retain stage to learn from the new experience. Case-based planning (CBP) is the idea of planning as remembering [10]. CBP is a specialization of case-based reasoning (CBR) which is a problem solving methodology based on using a library of solutions for similar problems [10]. In CBP, the solution proposed to solve a given problem is a plan, so this solution is generated taking into account the plans applied to solve similar problems in the past. The problems and their corresponding plans are stored in a plans memory. Problem description (initial state) and solution (situation when final state is achieved) are represented as beliefs, the final state as a goal (or set of goals), and the sequences of actions as plans. The CBP cycle is implemented through goals and plans. When the goal corresponding to one of the stages is triggered, different plans (algorithms) can be executed concurrently to achieve the goal or objective. Each plan can trigger new sub-goals and, consequently, cause the execution of new plans.

Hybrid CBR-BDI and CBP-BDI agents are supported by BDI agents that manage a set of technologies to obtain all the context information required by the reasoning and planning mechanisms implemented, creating AmI-based systems that automatically adapt themselves to the changes in the environment.

\section{Technologies for Context Awareness}

The essential aspect in this work is the development of an AmI-based architecture as the core of multi-agent systems over automated and dynamic environments. Thus the use of technologies that provide the agents automatic and real time information of the context, and allow them to react upon it, is also important. Ambient Intelligence (AmI) provides an effective way to create systems with the ability to adapt themselves to the context and users necessities. The vision of AmI assumes seamless, unobtrusive, and often invisible but also controllable interactions between humans and technology. AmI provides new possibilities for resolving a wide range of problems and proposes a new way to interact between people and technology, where this last one is adapted to individuals and their context, showing a vision where people are surrounded by intelligent interfaces merged in daily life objects [8], creating a computing-capable environment with intelligent communication and processing to the service of people by means of a simple, natural, and effortless human-system interaction for users [16]. One of the most benefited segments of population with the appearance of AmI-based systems will be the elderly and people with disabilities, improving important aspects of their life, especially health care [8]. 
RFID technology is a wireless communications technology used to identify and receive information about humans, animals and objects on the move. An RFID system contains basically four components: tags, readers, antennas and software. Tags with no power system (i.e. batteries) integrated are called passive tags or "transponders", these are much smaller and cheaper than active tags (power system included), but have shorter read range. Figure 1 shows how these four elements combined enable the translation of information to a user-friendly format. The transponder is placed on the object itself (i.e. bracelet). As this object moves into the reader's capture area, the reader is activated and begins signalling via electromagnetic waves (radio frequency). The transponder subsequently transmits its unique ID information number to the reader, which transmit it to a device or a central computer where the information is processed and showed. This information is not restricted to the location of the object, and can include specific detailed information concerning the object itself. It is used in various sectors including health care [18]. The configuration presented in this paper consists of a transponder mounted on a bracelet worn on the users' wrist or ankle, and several sensors installed over protected zones, with an adjustable capture range up to 2 meters, and a central computer where all the information is processed and stored.

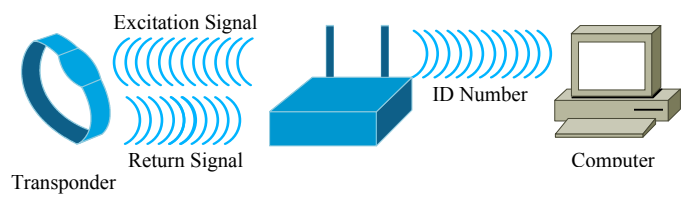

Fig. 1. Functioning of RFID technology.

Wireless LAN's (Local Area Network) also known as Wi-Fi (Wireless Fidelity) networks, increase the mobility, flexibility and efficiency of the users, allowing programs, data and resources to be available no matter the physical location [19]. These networks can be used to replace or as an extension of wired LANs. They provide reduced infrastructure and low installation cost, and also give more mobility and flexibility by allowing people to stay connected to the network as they roam among covered areas, increasing efficiency by allowing data to be entered and accessed on site. New handheld devices facilitate the use of new interaction techniques, for instance, some systems focus on facilitating users with guidance or location systems [15] by means of their wireless devices. The architecture presented in this paper incorporates "lightweight" agents that can reside in mobile devices, such as cellular phones, PDA's, etc. [2], and therefore support wireless communication, which facilitates the portability to a wide range of devices.

Automation devices are successfully applied on schools, hospitals, homes, etc. [13]. There is a wide diversity of technologies that provide automation services, one of them is ZigBee, a low cost, low power consumption, two-way, wireless communication standard, developed by the ZigBee Alliance [21]. It is based on IEEE 802.15 .4 protocol, and operates at $868 / 915 \mathrm{MHz} \& 2.4 \mathrm{GHz}$ spectrum. ZigBee is designed to be embedded in consumer electronics, , PC peripherals, medical sensor applications, toys and games, and is intended for home, building and industrial automation purposes, addressing the needs of monitoring, control and sensory 
network applications [21]. ZigBee allows star, tree or mesh topologies, and devices can be configured to act as (Figure 2): network coordinator (control all devices); router/repeater (send/receive/resend data to/from coordinator or end devices); and end device (send/receive data to/from coordinator).

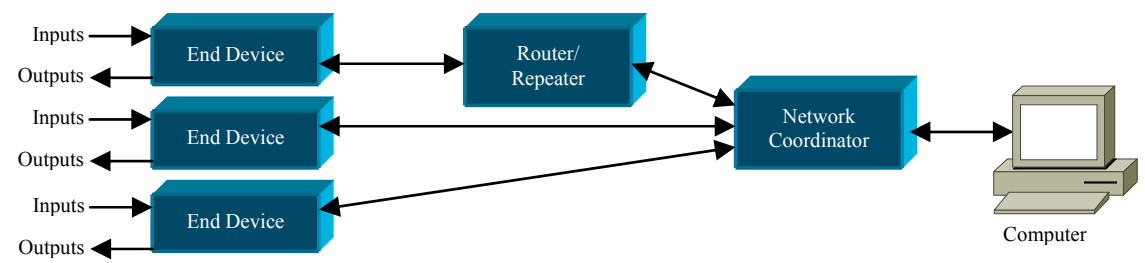

Fig. 2. ZigBee devices' configuration.

\section{Architecture Model}

Reasoning and planning mechanism, and the technology described before are integrated into a generic multi-agent system prototype that can be implemented on different scenarios (Figure 3), for example in geriatric residences [6], with some changes according the users and project necessities. The architecture implements five different agents, which have specific roles (Figure 4):

- User Agent is a BDI agent that runs on mobile devices (PDA). It manages the users' personal data and behaviour (monitoring, location, daily tasks, and anomalies). The beliefs and goals used for every user depend on the plan or plans defined by the super-users. User Agent maintains continuous communication with the rest of the system agents, especially with the ScheduleUser Agent (through which the scheduled-users can communicate the result of their assigned tasks) and with the SuperUser Agent. The User Agent must ensure that all the actions indicated by the SuperUser are taken out, sending a copy of its memory base (goals and plans) to the Manager Agent in order to maintain backups.

- SuperUser Agent is a BDI agent that runs on mobile devices (PDA). It inserts new tasks into the Manager Agent to be processed by the CBR mechanism. It also needs to interact with the User Agents to impose new tasks and receive periodic reports, and with the ScheduleUser Agents to ascertain plans' evolution.

- ScheduleUser Agent is a hybrid CBP-BDI planner agent that runs on mobile devices (PDA). It schedules the users' daily activities obtaining dynamic plans depending on the tasks needed for each user. It manages scheduled-users profiles (preferences, habits, holidays, etc.), tasks, available time and resources. Every agent generates personalized plans depending on the scheduled-user profile.

- Manager Agent is a hybrid CBR-BDI Agent that runs on a Workstation. It plays two roles: the security role that monitors the users' location and physical building status (temperature, lights, alarms, etc.) trough a continuous communication with the Devices Agent; and the manager role that handle the databases and the tasks assignation. It must provide security for the users and ensure the tasks assignments 
are efficient. This assignation is carried out through a CBR reasoning engine, which is incorporated within the Manager Agent. When a new assignation of tasks needs to be carried out, both past experiences and the needs of the current situation are recalled, allocating the respective and adequate task.

- Devices Agent is a BDI agent that that runs on a Workstation. This agent controls all the hardware devices. It monitors the users' location (continuously obtaining/updating data from the RFID readers), interact with the ZigBee devices to receive information and control physical services (lights, door locks, etc.), and also check the status of the wireless devices connected to the system (PDA's). The information obtained is sent to the Manager Agent to be processed.

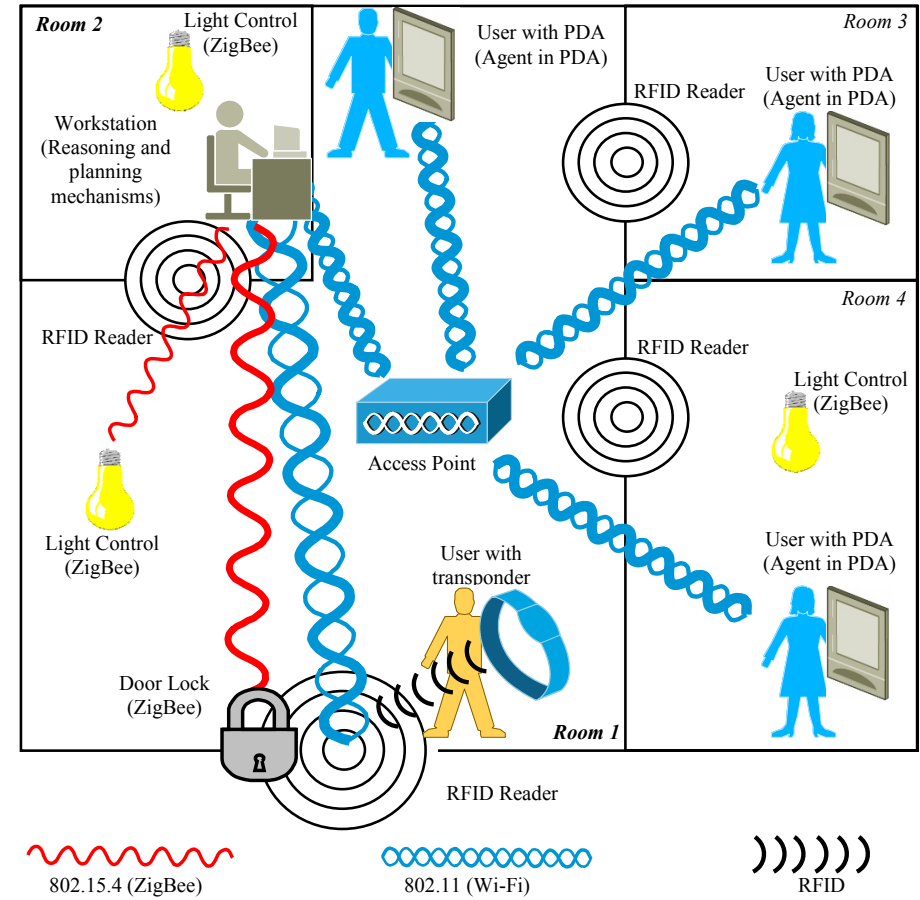

Fig. 3. Architecture applied on an automated environment.

The essential hardware used is: Sokymat's Q5 chip 125KHz RFID wrist bands and computer interface readers for people identification and location monitoring; Silicon Laboratories' C8051 chip-based 2.4GHz development boards for physical services automation (heating, lights, door locks, alarms, etc.); mobile devices (PDA's) for interfaces and users' interaction; a Workstation where all the high demanding CPU tasks (planning and reasoning) are processed; and a basic Wi-Fi network for wireless communication between agents (in PDA's and Workstation). All the hardware is some way integrated to agents, providing them automatic and real time information about the environment that is processed by the reasoning and planning mechanisms to automate tasks and manage physical services. 


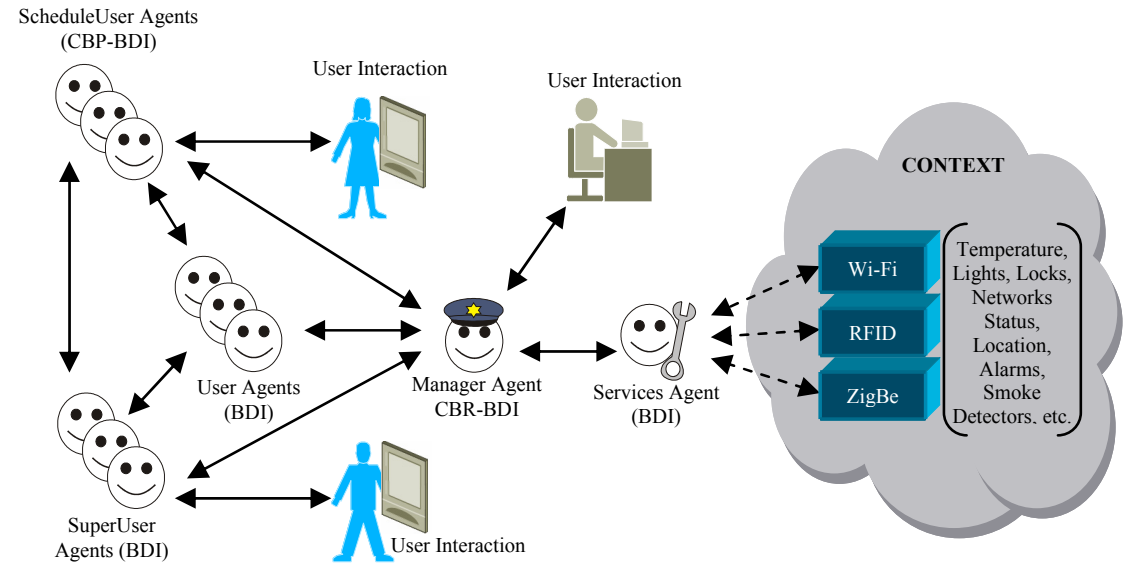

Fig. 4. Agents and technology in the architecture.

\section{Conclusions and Future Work}

Deliberative BDI agents with reasoning and planning mechanisms and the use of technology to perceive the context, provide a robust, intelligent and flexible architecture that can be implemented in wide variety scenarios, such as hospitals, geriatric residences, schools, homes or any dynamic environment where is a need to manage tasks and automate services. In fact, a prototype system, based on this architecture has been successfully implemented into a geriatric residence [6], improving the security and the health care efficiency through monitoring and automating medical staff's work and patients' activities, facilitating the assignation of working shifts and reducing time spent on routine tasks.

The use of CBR systems helps the agents to solve problems, adapt to changes in the context, and identify new possible solutions. These new hybrid agent models supply better learning and adaptation than pure BDI model. In addition, RFID, Wi-Fi and ZigBee devices supply the agents valuable information about the environment, processed trough reasoning and planning mechanisms, to create a ubiquous, noninvasive, high level interaction among users and the system.

However, it is necessary to continue developing and improving the AmI-based architecture presented, adding new capabilities and integrating more technologies to build more efficient and robust systems to automate services and daily tasks.

Acknowledgements. This work has been partially supported by the MCYT TIC200307369-C02-02 and the JCYL-2002-05 project SA104A05. Special thanks to Sokymat by the RFID technology provided and to Telefónica Móviles (Movistar) for the wireless devices donated. 


\section{References}

1. Allen, J.F.: Towards a general theory of action and time. Artificial Intelligence Vol. 23 pp. 123-154. (1984)

2. Bohnenberger, T., Jacobs, O., Jameson, A.: DTP meets user requirements: Enhancements and studies of an intelligent shopping guide. Proceedings of the Third International Conference on Pervasive Computing (PERVASIVE-05), Munich, Germany. (2005)

3. Bratman, M.E.: Intentions, Plans and Practical Reason. Harvard University Press, Cambridge, M.A. (1987)

4. Corchado J.M., Bajo J., de Paz Y. and Tapia D. I.: Intelligent Environment for Monitoring Alzheimer Patients, Agent Technology for Health Care. Decision Support Systems. Eslevier Science. DOI 10.1016/j.dss.2007.04.008. In Press (2007)

5. Corchado, J.M., Laza, R.: Constructing Deliberative Agents with Case-based Reasoning Technology. International Journal of Intelligent Systems. Vol. 18 No.12 1227-1241 (2003)

6. Corchado J.M., Bajo J., de Paz Y. and Tapia D. I.: Intelligent Environment for Monitoring Alzheimer Patients, Agent Technology for Health Care. Decision Support Systems. Eslevier Science. DOI 10.1016/j.dss.2007.04.008. In Press (2007)

7. Ducatel, K., Bogdanowicz, M., Scapolo, F., Leijten, J., Burgelman, J.C.: That's what friends are for. Ambient Intelligence (AmI) and the IS in 2010. Innovations for an eSociety. Challenges for Technology Assessment. Berlin, Germany. (2001)

8. Emiliani P.L., Stephanidis, C.: Universal access to ambient intelligence environments: opportunities and challenges for people with disabilities. IBM Systems Journal. (2005)

9. Glez-Bedia, M., Corchado, J.M.: A planning strategy based on variational calculus for deliberative agents. Computing and Information Systems Journal. Vol.10(1) 2-14. (2002)

10. Hammond, K.: Case-Base Planning: Viewing Planning as a Memory Task. Academic Press, New York. (1989)

11. Leung K.S., Jin H.D., Xu Z.B.: An expanding Self-organizing Neural Network for the Traveling Salesman Problem. Neurocomputing, vol. 62. (2004). pp 267-292.

12. Kolodner J.: Case-based reasoning. Morgan Kaufmann (1993).

13. Mainardi, E., Banzi, S., Bonfè, M. \& Beghelli, S. (2005). A low-cost Home Automation System based on Power-Line Communication Links. 22nd International Symposium on Automation and Robotics in Construction ISARC 2005. September 2005. Ferrara, Italy.

14. Pokahr, A., Braubach L., Lamersdorf, W.: Jadex: Implementing a BDI-Infrastructure for JADE Agents, in: EXP - In Search of Innovation (Special Issue on JADE), Vol. 3, 76-85. Telecom Italia Lab, Turin, Italy, September (2003)

15. Poslad, S., Laamanen, H., Malaka, R., Nick, A., Buckle, P., Zipf, A.: Crumpet: Creation of user- friendly mobile services personalised for tourism. In Proceedings of 3G. (2001)

16. Richter, K., Hellenschmidt, M.: Interacting with the Ambience: Multimodal Interaction and Ambient Intelligence. Position Paper to the W3C Workshop on Multimodal Interaction, 19-20 July. (2004)

17. Schmidt, A.: Interactive Context-Aware Systems Interacting with Ambient Intelligence. In G. Riva, F. Vatalaro, F. Davide \& M. Alcañiz, Ambient Intelligence, IOS Press pp. 159178. (2005)

18. Sokymat.: Sokymat. http://www.sokymat.com. (2006)

19. Sun Microsystems. (2000). Applications for Mobile Information Devices. Helpful Hints for Application Developers and User Interface Designers using the Mobile Information Device Profile. Sun Microsystems, Inc.

20. Susperregi, L., Maurtua, I., Tubío, C., Pérez M.A., Segovia, I., Sierra, B.: Una arquitectura multiagente para un Laboratorio de Inteligencia Ambiental en Fabricación. 1er. Taller de Desarrollo de Sistemas Multiagente (DESMA). Málaga, España. (2004)

21. ZigBee Standards Organization.: ZigBee Specification Document 053474r13. ZigBee Alliance. (2006) 